\title{
Evaluation of Automatic Vehicle Location Technologies for Paratransit in Small and Medium-Sized Urban Areas
}

\author{
Gary S. Spring \\ North Carolina A\&T State University \\ John Collura \\ Kenneth B. Black \\ University of Massachusetts
}

\begin{abstract}
This paper presents an evaluation framework designed for small and mediumsized city automatic vehicle location (AVL)-based paratransit systems and describes the application of that framework to one such paratransit system in North Carolina. Issues addressed include the benefits of implementing AVL, whether AVL for paratransit can be used effectively in small to medium-sized cities, and the problems associated with implementing such technologies. The paper is meant to provide guidance for other similar AVL evaluation efforts. A controlled before and after study approach is used to evaluate the AVL system in the City of Winston-Salem, North Carolina (population 265,878). Key findings are that the paratransit system's efficiency improved slightly with AVL but that demand variables did not change significantly relative to non-AVL equipped vehicles. Reasons for this outcome are examined.
\end{abstract}




\section{Introduction}

\section{Background}

Intelligent Transportation Systems (ITS) include a range of technologiessuch as automatic vehicle location systems (AVL), on-board guidance systems, electronic payment systems, automated control systems, and crash avoidance systems. These technologies hold great promise for increasing operational efficiency and safety of public transit systems. The Americans with Disabilities Act of 1990 includes requirements for complementary paratransit systems that can respond to previous-day reservations and that could lead to provision of realtime scheduling and dispatch. Operational pressures, such as vehicles operating at 25 percent or lower capacity, and subsidized costs of carrying passengers that sometimes exceed twice the fare of comparable taxi service are additional indicators of existing inefficiencies.

In response to this need, the Federal Transit Administration (FTA) has made the applications of ITS technologies to public transit a priority through its Advanced Public Transportation Systems (APTS) program. As with ITS in the highway arena, the application of APTS technologies offers potential in improved operational efficiency, lower costs, and enhanced system effectiveness. This paper presents an evaluation framework designed to assist in the assessment of small and medium-sized city AVL-based paratransit systems. The paper illustrates the application of the framework using one such paratransit system in Winston-Salem, North Carolina. The design and application of the framework are consistent with FTA's APTS Evaluation Guidelines (Casey and Collura 1993) and will be of interest to transit planners and managers in small and mediumsized cities. It is hoped that this information will be useful as a guideline for other similar efforts.

\section{Automatic Vehicle Location (AVL)}

As the name implies, automatic vehicle location devices provide location information about vehicles automatically. There are several different approaches 
for accomplishing this, some quite old (land-based systems) and some fairly new (satellite-based systems).

\section{Land-Based Systems}

Land-based AVL technology has been used by railroads in some form since the late 19th century, whereas the application of AVL to local transit began in the 1980s. These systems are of limited use for variable route, demand response systems, however, because they cannot track vehicles that are not on course, with the exception of Loran $C$ where topography permits, as explained below. The most common types of systems include dead reckoning, Loran $\mathrm{C}$, sign post and radio/cell phone. A brief description of each is provided below.

Dead Reckoning. Probably the oldest of the location methods, dead reckoning was the navigational system used by early sailors to determine location on the open sea. It involves traveling in a known direction at a known speed for a measured time interval (on transit systems, the odometer is used for distance determination rather than the speed and time measures). Given the distance traveled, the direction traveled and the position of the starting point, a new location can be calculated. The method has serious limitations. The first is that errors accumulate. Small errors in each leg of a trip result in significant positional errors at the final destination-thus requiring that accurate intermediate locations be established along the route. Second, accurate distance measurements are critical. Yet, the accuracy of the accumulated mileage on a bus route is only as accurate as the bus odometer which is on the average around $+/-2$ percent (100 feet on a 20-mile bus route). Third, accurate direction is difficult to obtain. Usually, the magnetic compass in the bus must be initialized and is easily influenced by local attraction (NIACAD 1991).

Loran $C$. Originally developed for maritime applications, Loran-C is a long range, signal-triangulation navigation system. It operates using a system of government-run radio transmission sources and was initially developed for use by ships and aircraft. Radio signals are received from three or more transmitters whose locations are known. The location of the receiver can be calculated using triangulation techniques. The transmitters must be in the direct line of sight of 
the receivers; therefore, Loran- $C$ positioning is severely limited by topography. Given this limitation and the fact that the technology is being phased out with the advent of global positioning systems (GPS), it is, again, of limited use for transit applications (Smith 1990).

Sign Post. The railroad industry has used sign post technology for years. The technology consists of devices along fixed routes that recognize when a vehicle passes them and communicates that information to a central dispatching office. Although expensive, due to the significant amount of infrastructure required, sign post technology has been used for fixed route transit. It is not, however, applicable to demand response systems such as paratransit because of the fixed nature of the infrastructure (Labell 1992).

Terrestrial Radio/Cell Phone. These consist of a set of fixed receivers that receive signals from a vehicle's radio or cell phone and subsequently calculate the vehicle's position by triangulation. This requires that specialized infrastructure be built. It is of limited use for variable route systems due, again, to the fixed infrastructure requirement.

\section{Satellite-Based Systems}

The most common type of satellite-based systems is the global positioning system. A brief description is provided below.

Global Positioning Systems (GPS). A space-based system of artificial satellites and ground receivers, global positioning systems provide location in three dimensions. The system currently has 27 satellites in place. Receivers obtain signals from four or more satellites to calculate their position using triangulation. The system was created and originally used by the U.S. Air Force Space Command. It is now being used extensively for civilian applications. Due to the selective availability of the satellites, location accuracy using affordable receivers is around 50 to 100 feet-certainly adequate for AVL transit applications (Kavanagh 1996). The more expensive differential GPS systems, providing accuracies up to the nearest centimeter, are not necessary for this application. 


\section{Description of the Winston-Salem Paratransit System}

The Winston-Salem Transit Authority (WSTA), with more than 150 vehicles, provides fixed-route, modified fixed-route, downtown circulation, fringe park and shuttle, park and ride, demand responsive paratransit, contract paratransit, vanpools, carpool matching, and vehicle brokerage services. WSTA serves the City of Winston-Salem and surrounding counties.

Trans-AID is WSTA's paratransit, dial-a-ride service and serves the city of Winston-Salem and Forsythe County (1990 population 265,878). Trans-AID serves approximately 800 passengers per day with its 17 paratransit vehicles and currently runs a geographic information systems (GIS) based automated scheduling and dispatching system (Stone et al. 1996). WSTA, along with the North Carolina Department of Transportation, FTA and others, funded a Mobility Management Project whose purpose is to "provide human service agency passengers, commuters, and the general public with 'one stop shopping' for multimodal public transportation" (Stone et al. 1996). The potential of GIS/AVL for intermodal connections was not examined as part of the study since it was beyond the study's scope. WSTA wants this new system to serve as a test-bed for evaluating advanced technologies. As part of a pilot study, it purchased GPS units, mobile data terminals (MDT) and smart cards for three of its paratransit vehicles. The equipment was installed at the beginning of June 1995.

\section{Description of AVL-Based Paratransit Systems}

There are a great number of transportation applications for which AVL may be (and often is) profitably applied, including emergency response, truck and bus fleet management, and paratransit systems. The generic AVL-based paratransit system consists of a device to determine a vehicle's location, some sort of communication device in the transit vehicle and in the dispatch office, GIS software to display location information, and scheduling and dispatching software to provide the capability to use the location information.

WSTA's system uses GPS for location, MDTs and radio for communication, and a MapInfo-based scheduling and dispatching software package (PASS). Radio communication is accomplished using a radio "trunking" system, which 
is essentially a "time share" of air space and, thus, does not require purchase of a dedicated radio frequency. For the purposes of this paper, the term AVL is used to represent this combination of equipment.

\section{Rationale and Significance of Study}

\section{Problem Statement}

Central to the ability of APTS to improve paratransit efficiency is the improvement of the process of dispatching and scheduling demand-responsive transportation. The manner in which vehicles and trips are matched directly affects passenger service levels and system productivity. Locating shared-ride transit vehicles in real time is key to success in this regard. At the beginning of a typical paratransit tour, a pre-arranged schedule is given to each driver which lists the sequence, pickup times, addresses and destinations of its passengers as well as directions for minimum time or distance paths to follow. This scenario does not lend itself to real-time operation. Using automatic vehicle location (AVL) technology, each vehicle's location is known at any point in time and, with the integration of GIS technology, may be displayed on a map display at the control center. The AVL, along with the GIS database linked to customer information would facilitate real time requests for service-perhaps, initially, providing sameday service, gradually approaching real-time service. The AVL/GIS would allow insertions for "will calls" (unscheduled return trips) to be made more efficiently as well. Additionally, with in-vehicle displays (such as MDTs), the driver's schedule may be stored in digital form and could be updated as necessary.

\section{Advantages of AVL-Based Paratransit}

The potential advantages of AVL vis á vis paratransit, in terms of increased operational efficiency, effectiveness and safety can be significant. Real-time information about vehicle location would:

- provide the capability to coordinate paratransit with fixed route facilities-thus reducing operating costs;

- allow same-day scheduling, approaching real-time response;

- $\quad$ provide improved service potential (more timely, more flexible, etc.); 
- enhance driver and passenger safety/security; and

- provide information for personnel management programs.

Of course, effective use of the AVL-provided information requires a graphical display such as that provided by geographic information systems. This combination of technologies offers many potential benefits to paratransit operators. In the near term, operators would be able to observe system performance in real time and make immediate adjustments where necessary, potentially avoiding service delays. Service could then be more responsive to travelers' demands rather than scheduled a day or more in advance. The potential for improved efficiency of scheduling and dispatching using the AVL/GIS-provided information would provide better and less expensive service. This would encourage increased ridership, thus increasing operational effectiveness of the system as well. Another, somewhat less tangible but nevertheless very real, benefit would be the capability of sending assistance to the location of an emergency signal transmission which could save lives where voice communication may not be possible. For the long term, knowledge-based decision support systems would observe system performance and respond more quickly and efficiently than the human operator is currently able, thus improving operational efficiency even more.

These advantages are well recognized and many paratransit properties in the United States are planning implementation of an AVL system in the very near future (Spring et al. 1995). There exists, therefore, a critical need for a methodology to evaluate these systems.

A survey of paratransit properties across the U.S. (Spring et al. 1995) regarding AVL-based paratransit evaluation found that only one system, Houston's MetroLift, has progressed far enough in its implementation of AVL on paratransit to be able to conduct an evaluation. That system is a relatively large one and serves a major city.

The potential advantages of AVL-based paratransit, coupled with the fact that no other efforts have been made to evaluate an AVL-based paratransit system in a small or medium-sized city, have shaped the objective for this paper, 
which is to develop and illustrate the use of an evaluation framework to assess AVL systems on paratransit services in small and medium-sized urban areas.

\section{Research Approach}

The general approach taken for the Trans-AID evaluation was that of the pilot study, where a subset of information was used to demonstrate how a new technology may be used to solve a particular problem or problems and to identify implementation problems. The City of Winston-Salem was chosen as the pilot site for several reasons:

- It had already implemented scheduling and dispatching software.

- It was willing and able to serve as a test bed.

- It had requisite software (PASS, which is a GIS-based automated scheduling and dispatching package), and had AVL units on three of its paratransit buses.

Key study questions of interest in the evaluation were:

- What are the benefits of implementing AVL?

- Can AVL for paratransit be used effectively in small to medium-sized cities?

- What are the problems associated with implementing such technologies?

\section{Experimental Design}

To evaluate the effectiveness of AVL operation, a "before and after" study was performed on the WSTA system in which non-AVL operation (before period) was compared to AVL operation (after period) using a set of efficiency and effectiveness evaluation criteria. There are several serious disadvantages to the standard before and after study (Hummer 1994), which were addressed as part of the current study's experimental design. First, after "treatment" (in this case, installation of the AVL units) there exists a period of adjustment during which data should not be used. AVL units were installed in May 1995 on three WSTA paratransit vehicles. Data for the "after" period began in September 1995, which allowed in excess of three months for the adjustment period. A second disadvantage to the approach concerns changes in evaluation measures caused by factors 
other than the treatment (Council et al. 1980). This was addressed in two ways: both the "before" and "after" periods were selected during the same time of year (September-October time frame); and, the "before period" was chosen so that it fell well after implementation of scheduling and dispatching software but before installation of the AVL. The third, and most difficult, is the so-called "maturation" problem - that is, changes in evaluation measures may be due to pre-existing trends in the variables used. The second and third issues were addressed using a "controlled" experiment. Given that WSTA chose to install AVL on only three of its 17 vehicles, it was possible to perform a controlled experiment using a control group (the non-AVL equipped vehicles) and a treatment group (AVLequipped vehicles). Percent changes in measures rather than absolute changes from before and atter were compared. For example, the percent change in numbers of passenger trips per vehicle hour from the "before" to the "after" period was calculated for both the control and treatment groups. The "hours of service" variable was calculated using elapsed time from first pick up of the day to the last drop off. These changes were statistically tested to determine whether they were significantly different from one another. Figure 1 depicts the situation.
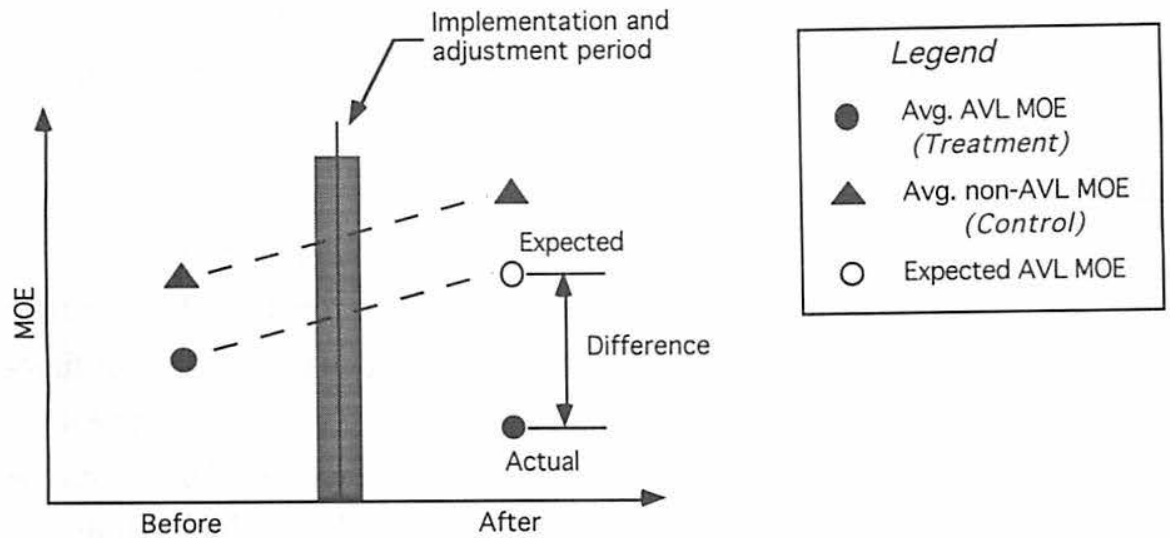

Figure 1. "Before" and "after" with control. 


\section{Data Collection}

The City of Winston-Salem's current paratransit activities were characterized. Details regarding current hardware and software capabilities, personnel, bus fleet, ridership figures, driver logs, and so on, were collected as part of this task. Data from the PASS-generated driver log files were used in developing measures of effectiveness (MOE) for the project. Each day, a driver log dBase file with 65 fields was generated by the PASS software. For the "before" period, data from files for October 3, 1994, through October 31, 1994, were used to create MOE values. Only weekday operations were considered. For the "after" period, data from files for September 11, 1995, through October 6, 1995, were used. Each data file contains approximately 900 records, one for each passenger trip served that day. Variables extracted from the files are:

RIDERS

CAPICALC

CAPCALC

COLLECT

NEGETA
Number of passenger trips for the day

Number of passengers with special needs

Bus occupancy

Time of day that driver actually completed pick up or drop off

Negotiated pick up/drop off time of day

Aggregate values of these variables were calculated for each day and entered into a summary file. Each day, representing between 800 and 900 entries, constitutes one record in the summary file.

\section{Evaluation Framework}

The FTA guidelines for evaluating advanced technologies mentioned earlier (Casey et al. 1993) were used as a guide for this evaluation. Measures of effectiveness were identified in an attempt to capture as many of the four functional areas defined in the APTS Guidelines as possible. Figure 2 lists the MOEs used and indicates in which functional evaluation area each lies. Data from the PASS-generated driver log files were used to calculate MOE values. MOE values, for "before" and "after" implementation of the AVL, were used for evaluation. 


\begin{tabular}{|c|c|c|c|c|c|}
\hline \multirow[b]{2}{*}{ Name } & \multirow[b]{2}{*}{ Description } & \multicolumn{4}{|c|}{$\begin{array}{c}\text { Performance } \\
\text { Dimension }\end{array}$} \\
\hline & & 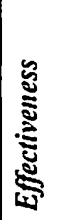 & 忥 & : & 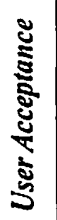 \\
\hline Pass Trips & $\#$ of passenger trips (per day) & $\checkmark$ & & & $\checkmark$ \\
\hline SP Needs Patrons & \# of patrons with special needs served & $\checkmark$ & & & \\
\hline Bus Occupancy & Bus occupancy & $\checkmark$ & & & \\
\hline Pass Trips per Veh-Hr & \# of passenger trips per vehicle hour & $\checkmark$ & & & \\
\hline Time Deviation & pickup time agreed vs pickup time provided & & $\checkmark$ & & \\
\hline
\end{tabular}

Figure 2. Evaluation matrix.

\section{Discussion of Results}

All variables were tested for normality. It was found that there is not enough statistical evidence at the 95 percent confidence level to say that they are not normally distributed. Table 1 provides a summary of normality test results. However, the type of test to which the variables were subjected is a "robust" test

\begin{tabular}{|lccc|}
\hline \multicolumn{4}{c|}{$\begin{array}{c}\text { Table 1 } \\
\text { Tests for Normality }\end{array}$} \\
\hline \multicolumn{1}{|c}{ Variable } & $\chi^{2}$ & $\chi^{2}$ Critical & Result \\
\hline Passenger Trips & 48.52 & 51.00 & Normal \\
Special Needs Patrons & 26.05 & 31.41 & Normal \\
Bus Occupancy & 98.54 & 101.88 & Normal \\
Passenger Trips/Vehicle Hour & 13.60 & 26.30 & Normal \\
Time Deviation & 37.45 & 59.30 & Normal \\
\hline
\end{tabular}


(Kachigan 1982) and, therefore, the assumption of normality for all variables was a valid one.

Entries in Table 2 were calculated in the following ways:

- $\% \Delta$ for nonAVL and AVL groups was calculated by subtracting the average "before" for the individual parameter from its respective "after" value and dividing by the "before" value. Equation 1 illustrates the process:

$$
\% \Delta_{A V L \text {-or-nemAVL }}=\frac{\chi_{\text {Afler }}-\chi_{\text {Befine }}}{\chi_{\text {Befine }}} \times 100 \%
$$

Eq. 1

\begin{tabular}{|c|c|c|c|c|c|c|}
\hline \multicolumn{7}{|c|}{$\begin{array}{c}\text { Table } 2 \\
\text { Summary of Results }\end{array}$} \\
\hline & & Trips & $\begin{array}{c}\text { Special } \\
\text { Needs } \\
\text { Patrons }\end{array}$ & \multicolumn{2}{|r|}{$\begin{array}{c}\text { Trips } \\
\text { per } \\
\text { Veh. Hr. }\end{array}$} & $\begin{array}{l}\text { Time } \\
\text { Dev. }\end{array}$ \\
\hline \multirow[t]{2}{*}{ NonAVL } & $\% \Delta$ & $40 \%$ & $28 \%$ & $28 \%$ & $55 \%$ & $76 \%$ \\
\hline & $\mathbf{s}$ & $20 \%$ & $30 \%$ & $28 \%$ & $31 \%$ & $69 \%$ \\
\hline \multirow[t]{2}{*}{ AVL } & $\% \Delta$ & $-24 \%$ & $-18 \%$ & $-17 \%$ & $-13 \%$ & $26 \%$ \\
\hline & $\mathbf{s}$ & $61 \%$ & $64 \%$ & $59 \%$ & $74 \%$ & $102 \%$ \\
\hline \multirow[t]{2}{*}{ Difference } & $\% \Delta$ & $-63 \%$ & $-46 \%$ & $-45 \%$ & $-67 \%$ & $-50 \%$ \\
\hline & $s_{e}$ & $15 \%$ & $17 \%$ & $16 \%$ & $19 \%$ & $30 \%$ \\
\hline \multirow[t]{2}{*}{ Hypotheses: } & $t$ & -4.08 & -2.68 & -2.84 & -3.45 & -1.66 \\
\hline & $t_{90}$ & -1.75 & -1.75 & -1.75 & -1.75 & -1.34 \\
\hline \multirow[t]{2}{*}{ Ho: $A V L=$ NonAVL } & $t_{95}$ & -2.13 & -2.13 & -2.13 & -2.13 & -1.75 \\
\hline & $t_{99}$ & -2.95 & -2.95 & -2.95 & -2.95 & -2.60 \\
\hline \multirow[t]{3}{*}{ Alternatives: } & AVL & AVL & AVL & AVL & AVL & AVL \\
\hline & $<$ & $<$ & $<>$ & $<>$ & $<$ & $<$ \\
\hline & AVL & NonAVL & NonAVL & NonAVL & NonAVL & NonAVL \\
\hline
\end{tabular}


- Standard deviations for the two groups were calculated using equation 2 shown below (Kachigan 1982).

$$
\mathrm{s}=\sqrt{\mathrm{s}_{A}^{2}+\mathrm{s}_{A}^{2}-2 \mathrm{rs}_{A} \mathrm{~s}_{B}}
$$

where:

$\mathrm{S}_{A}=$ standard deviation of the "after" period

$\mathrm{s}_{B}=$ standard deviation of the "before" period

$r=$ the correlation coefficient between the "before" and "after" groups.

- $\quad \% \Delta$ for the "Difference" group was calculated by subtracting the nonAVL $\% \Delta$ from the AVL $\% \Delta$. Equation 3 illustrates the process:

$$
\% \Delta=\% \Delta_{A V L}-\% \Delta_{n u n A V L}
$$

The standard error term for the above difference value was calculated using equation 4 , shown below:

$$
s_{x / c}^{2}=(1 / c)^{2} s_{x}^{2}
$$

where $c=\bar{\chi}_{\text {Before }}$ used in equation 1 .

Each MOE was subjected to the following hypothesis test:

Ho: $\% \Delta=0$

Ha: $\% \Delta \neq 0$ (for Time Deviation: $\% \Delta<0$ )

Therefore, all were 2-tail tests with the exception of Time Deviation. The Student's t-distribution was used for the tests with 15 degrees of freedom (each group had 17 observations). The t statistic was calculated by dividing $\% \Delta$ by the 
$\mathrm{s}_{\mathrm{e}}$ value. Critical values for 90 percent, 95 percent and 99 percent confidence levels are shown in Table 2.

Table 2 indicates that neither non-AVL-equipped nor AVL-equipped vehicles changes in the "before" and "after" periods are significantly different from 0 (all $t$ values, although not shown, are less than the critical $t$ for 95 percent) yet differences in percent change from before and after periods are significant for all MOEs.

The time deviation MOE, an efficiency measure, shows the AVL equipped buses performed better (at the 90 percent confidence level)-i.e., the reduction in time deviation is for those vehicles significantly greater than that for the nonAVL-equipped vehicles. This is not surprising, given the nature of AVL. The dispatcher is better able to do insertions, for example, which would reduce time deviations.

A reasonable explanation, perhaps, for why the AVL showed no improvement in the demand-related effectiveness variables, yet did indicate improvement for the efficiency MOE, is that the AVL would need to be implemented on the whole system before the improvements in service could be expected to affect demand. Users may not perceive the improvements in efficiency, especially if the same buses are not used consistently by the same patrons.

\section{Conclusions and Recommendations}

The City of Winston-Salem's Trans-AID system, as part of a pilot study, installed and implemented AVL devices on three of its 17 vehicles. The system's MDT-AVL equipment was evaluated using a "before and after" experimental design. Findings indicate that the AVL devices have led to an improvement in the efficiency performance measure (time deviation). None of the effectiveness or user acceptance measures showed any statistically significant improvement over the non-AVL-equipped vehicles. It was postulated that this may be due to the short time during which the AVL has been implemented and to the fact that only three vehicles were AVL-equipped. It seems reasonable that demand-related measures would not improve unless all vehicles were so equipped and a longer time were observed. 


\section{Major Conclusions}

The evaluation framework may be used by small and medium-sized city paratransit systems for their AVL systems to answer several questions, including those posed at the outset:

- What are the benefits of implementing AVL?

- Can AVL for paratransit be used effectively in small to medium-sized cities?

- What are the problems associated with implementing such technologies?

Costs and Benefits of Implementing AVL. Not surprisingly, an outcome of this study indicates that operation efficiency can improve with the use of AVL, even with minimal use of AVL technology (only 3 of 17 vehicles were equipped with AVL equipment and the GPS portion of the AVL was used only minimally for insertions, although MDTs were used extensively). This can result in reductions in deadhead miles that may allow the operator to provide more service. Other potential benefits which might follow are demand-related, such as increased ridership and occupancy. These measures did not increase as expected, as stated earlier, perhaps because the AVL devices were not implemented on a wide enough basis (i.e., on the whole system).

Although initial equipment and set up costs can be high $(\$ 15,000)$, per-unit costs are only about $\$ 3,000$ for each vehicle and are expected to come down. These per vehicle costs include mobile data transfer units, GPS units, modems and software. This does not include the cost of a dedicated radio frequency, which is quite substantial. This, however, given recent advances in "piggy-backing" technology, may not be an issue in the near future.

Effectiveness of AVL for Paratransit for Small to Medium-sized Cities. Although the demand-related measures normally associated with effectiveness showed no improvement, it may be concluded that AVL use did improve the effectiveness of the WSTA paratransit system. Effectiveness means that the system has attained a desired effect. One desired effect of a paratransit system is improved service to its riders, whether or not they are able to perceive that im- 
provement. The system's service did improve significantly, as evidenced by the changes in time deviation.

Problems Associated with Implementing Such Technologies. AVL requires that several issues be resolved for its successful implementation. First, it requires a radio frequency for communicating vehicle positions from the MDT in the vehicle to the dispatcher. The WSTA system used a trunking system, which is essentially a time-sharing system with other users of the radio frequency. Access to the frequency is therefore limited, which could lead to positional accuracy problems. For example, if polling is limited to every three minutes, a vehicle could easily travel one to two miles from the position relayed. Second, users (passengers and clients) must perceive that they are reaping benefits from the use of AVL, and drivers and dispatchers must accept and use the AVL devices. Third is a study design issue. Although equipping a small subset of vehicles introduces problems, the controlled approach explained here is superior to the standard "before and after" study. The extreme changes in variables from before to after in the control (unequipped) group, described in Table 2, support this statement.

\section{Recommendations}

Several recommendations can be derived from this study. First, and perhaps most important, is the evaluation study design used, that is, a controlled "before and after" design. The controlled experiment examines differences in changes from before to after for each group. It therefore accounts for changes in evaluation measures that may have been due to pre-existing trends in the variables used or other outside influences on the system that may cause system changes in performance. Presumably, the control and treatment groups are both subject to these trends/influences. One would therefore expect that changes in their performance variables would not be significantly different-statistically speaking. Of course, this study was limited, in that only three of 17 vehicles were equipped with AVL, and no control was imposed on them in terms of service area. A better design would include a longer time frame, perhaps half the vehicles equipped with AVL, and more control over each group's service area — thus providing more 
consistent before and after data. It is recommended that future evaluations of AVL for paratransit adopt the controlled experiment approach for the reasons outlined previously. Second, the type of location technology recommended for paratransit operation is the satellite-based GPS. Reasons for this include:

- Paratransit's routes continually change, suggesting that the location system should not require a fixed, land-based infrastructure to operate.

- In small to medium-sized urban settings, GPS is typically more reliable than in large urban settings, where tall buildings sometimes create problems.

- The locational accuracy required is provided by the lower-end GPS units, so costs are less an issue.

- GPS does not require infrastructure other than the satellites already in place. The satellites are accessed free of charge and require no maintenance on the part of the agency.

- Finally, GPS is an emerging technology; it is anticipated that its use will continue to increase.

Third, it is suggested that a dedicated radio frequency is an extremely valuable characteristic of an AVL system. As indicated earlier, WSTA's trunking system is less than optimal. For example, for insertion purposes if polling occurs every three minutes, a vehicle could be 1.5 miles or more away. *

\section{Acknowledgment}

The authors thank Trans-AID staff at the Winston-Salem Transit Authority, whose active participation and amiable cooperation were key to the project's success. The authors also thank Dr. John Stone and Mr. Peter Gray-Mullen at North Carolina State University for their willingness to share data and ideas. This work was sponsored by the Urban Transit Institute at North Carolina A\&T State University. 


\section{References}

Casey R.F., and J. Collura. 1993. Evaluation Guidelines for the Advanced Public Transportation Systems Operational Tests, U.S. Department of Transportation, Federal Transit Administration, Report Number DOT-VNTSC-FTA-93-9, Washington D.C. Council F. M., et al. 1980. Accident Research Manual, U.S. Department of Transportation, Federal Highway Administration, Report Number FHWA/RD-80/016, Washington D.C.

Hummer J. E. 1994. Experiment Design, Manual of Transportation Studies, Appendix A, Institute of Transportation Engineers, Englewood Cliffs, NJ: Prentice-Hall. Kachigan S. K. 1982. Multivariate Statistical Analysis, New York: Radius Press.

NIACAD. 1991. AVL Technology Investigation and Applicability Report, prepared for PVTA, Ontario, Canada.

Kavanagh B. F. 1996. Surveying Principles and Applications, 4th Edition, Englewood Cliffs, NJ: Prentice Hall: 446-454.

Labell L. N., C. P. Schweiger, and M. Kihl. 1992. Advanced Public Transportation Systems: The State of the Art Update 1992, U.S. Department of Transportation, Federal Transit Administration, Report Number DOT-VNTSC-FTA-92-3.

Smith, T. 1990. A Guide to Loran Technology, Alpha Resource Inc (Company Literature), October: 76.

Spring G.S., J. Collura, and K. B. Black. 1995. Evaluation of Automated Vehicle Location Technologies Used In Automatic Scheduling \& Dispatching Systems for Small Urban Areas in North Carolina, Final Report to the Urban Transit Institute, North Carolina A\&T State University.

Stone J. R., and P. Gray-Mullen. 1996. Winston-Salem Mobility Management: An Evaluation of Computer-Aided Dispatch and Scheduling, Transportation Research Board, preprint for 1996 Annual Meeting.

\section{About the Authors}

Gary S. Spring is an Associate Professor in the Department of Civil Engineering at North Carolina A\&T State University.

John ColluRa is a Professor in the Department of Civil and Environmental Engineering at the University of Massachusetts, Amherst.

KenNeth BLaCK is a doctoral candidate in the Department of Civil and Environmental Engineering at the University of Massachusetts, Amherst. 